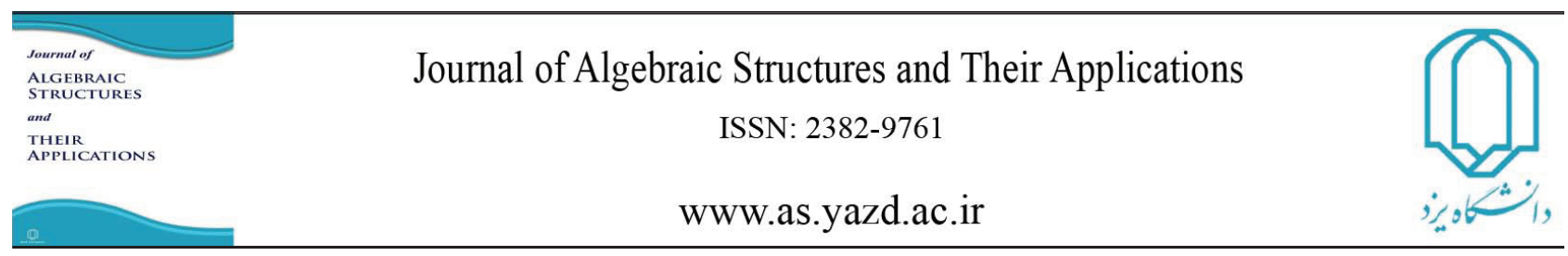

Algebraic Structures and Their Applications Vol. 6 No. 1 ( 2019 ) pp 99-102.

\title{
AN ELEMENTARY PROOF OF NAGEL-SCHENZEL FORMULA
}

\author{
ALIREZA VAHIDI
}

Abstract. Let $R$ be a commutative Noetherian ring with non-zero identity, $\mathfrak{a}$ an ideal of $R, M$ a finitely generated $R$-module, and $a_{1}, \ldots, a_{n}$ an $\mathfrak{a}$-filter regular $M$-sequence. The formula

$$
\mathrm{H}_{\mathfrak{a}}^{i}(M) \cong \begin{cases}\mathrm{H}_{\left(a_{1}, \ldots, a_{n}\right)}^{i}(M) & \text { for all } \mathrm{i}<\mathrm{n}, \\ \mathrm{H}_{\mathfrak{a}}^{i-n}\left(\mathrm{H}_{\left(a_{1}, \ldots, a_{n}\right)}^{n}(M)\right) & \text { for all } \mathrm{i} \geq \mathrm{n},\end{cases}
$$

is known as Nagel-Schenzel formula and is a useful result to express the local cohomology modules in terms of filter regular sequences. In this paper, we provide an elementary proof to this formula.

\section{INTRODUCTION}

Throughout $R$ will denote a commutative Noetherian ring with non-zero identity, $\mathfrak{a}$ and $\mathfrak{b}$ two ideals of $R, X$ an arbitrary $R$-module which is not necessarily finitely generated, and $M$ a finitely generated $R$-module. Recall that the $i$-th local cohomology functor $\mathrm{H}_{\mathfrak{a}}^{i}$ is the $i$-th right derived functor of the $\mathfrak{a}$-torsion functor $\Gamma_{\mathfrak{a}}$. For basic results, notations and terminology not given in this paper, the reader is referred to [T], [2], and [5].

DOI : $10.29252 /$ as. 2019.1359

MSC(2010): Primary: 13D45, Secondary: 14B15

Keywords: Filter regular sequences, local cohomology modules, Nagel-Schenzel formula.

Received: 25 Sep 2017, Accepted:20 April 2019.

This research was in part supported by a grant from Payame Noor University.

(C) 2019 Yazd University. 
The concept of an $\mathfrak{a}$-filter regular sequence is a generalization of the concept of a filter regular sequence which has been studied in [6] and [7], and has led to some interesting results. Let $a_{1}, \ldots, a_{n} \in \mathfrak{a}$. Recall that $a_{1}, \ldots, a_{n}$ is an $\mathfrak{a}$-filter regular $M$-sequence if

$$
\operatorname{Supp}_{R}\left(\frac{\left(a_{1}, \ldots, a_{i-1}\right) M:_{M} a_{i}}{\left(a_{1}, \ldots, a_{i-1}\right) M}\right) \subseteq \operatorname{Var}(\mathfrak{a})
$$

for all $1 \leq i \leq n$, where $\operatorname{Var}(\mathfrak{a})$ denotes the set of prime ideals of $R$ containing $\mathfrak{a}$. Let $a_{1}, \ldots, a_{n}$ be an $\mathfrak{a}$-filter regular $M$-sequence. Then, by [3, Proposition 1.2], we have

$$
\mathrm{H}_{\mathfrak{a}}^{i}(M) \cong \begin{cases}\mathrm{H}_{\left(a_{1}, \ldots, a_{n}\right)}^{i}(M) & \text { for all } i<n, \\ \mathrm{H}_{\mathfrak{a}}^{i-n}\left(\mathrm{H}_{\left(a_{1}, \ldots, a_{n}\right)}^{n}(M)\right) & \text { for all } i \geq n,\end{cases}
$$

which is known as Nagel-Schenzel formula. This formula was first obtained by Nagel and Schenzel, in [4, Lemma 3.4], in the case where $R$ is a local ring with maximal ideal $\mathfrak{m}$ and $\mathfrak{a}=\mathfrak{m}$. Both of them used the Grothendieck spectral sequence

$$
\mathrm{E}_{2}^{p, q}:=\mathrm{H}_{\mathfrak{a}}^{p}\left(\mathrm{H}_{\left(a_{1}, \ldots, a_{n}\right)}^{q}(M)\right) \underset{p}{\Longrightarrow} \mathrm{H}_{\mathfrak{a}}^{p+q}(M)
$$

to prove $(\mathbb{\square})$. In this paper, we provide an elementary proof to this formula.

\section{An elementary proof of (四)}

The following lemmas are needed in our proof of Nagel-Schenzel formula.

Lemma 2.1. Let $t$ be a non-negative integer such that $\mathrm{H}_{\mathfrak{a}}^{t-i}\left(\mathrm{H}_{\mathfrak{b}}^{i}(X)\right)=0$ for all $0 \leq i \leq t$. Then $\mathrm{H}_{\mathfrak{a}+\mathfrak{b}}^{t}(X)=0$.

Proof. We prove by using induction on $t$. The case $t=0$ is clear because $\Gamma_{\mathfrak{a}}\left(\Gamma_{\mathfrak{b}}(X)\right)=\Gamma_{\mathfrak{a}+\mathfrak{b}}(X)$. Suppose that $t>0$ and that $t-1$ is settled. Assume that $\bar{X}=X / \Gamma_{\mathfrak{b}}(X)$ and $Q=\mathrm{E}_{R}(\bar{X}) / \bar{X}$ where $\mathrm{E}_{R}(\bar{X})$ is an injective hull of $\bar{X}$. Since $\Gamma_{\mathfrak{b}}(\bar{X})=0=\Gamma_{\mathfrak{a}+\mathfrak{b}}(\bar{X}), \Gamma_{\mathfrak{b}}\left(\mathrm{E}_{R}(\bar{X})\right)=0=$ $\Gamma_{\mathfrak{a}+\mathfrak{b}}\left(\mathrm{E}_{R}(\bar{X})\right)$. Applying the derived functors of $\Gamma_{\mathfrak{b}}(-)$ and $\Gamma_{\mathfrak{a}+\mathfrak{b}}(-)$ to the short exact sequence

$$
0 \longrightarrow \bar{X} \longrightarrow \mathrm{E}_{R}(\bar{X}) \longrightarrow Q \longrightarrow 0
$$

we obtain the isomorphisms

$$
\mathrm{H}_{\mathfrak{b}}^{i}(Q) \cong \mathrm{H}_{\mathfrak{b}}^{i+1}(X)
$$

and

$$
\mathrm{H}_{\mathfrak{a}+\mathfrak{b}}^{i}(Q) \cong \mathrm{H}_{\mathfrak{a}+\mathfrak{b}}^{i+1}(\bar{X})
$$

for all $i \geq 0$. From the isomorphisms (घ), for all $0 \leqslant i \leqslant t-1$, we have

$$
\mathrm{H}_{\mathfrak{a}}^{(t-1)-i}\left(\mathrm{H}_{\mathfrak{b}}^{i}(Q)\right) \cong \mathrm{H}_{\mathfrak{a}}^{t-(i+1)}\left(\mathrm{H}_{\mathfrak{b}}^{i+1}(X)\right)
$$


which is zero by the assumptions. Thus, from the induction hypothesis on $Q$, we have $\mathrm{H}_{\mathfrak{a}+\mathfrak{b}}^{t-1}(Q)=0$. Therefore $\mathrm{H}_{\mathfrak{a}+\mathfrak{b}}^{t}(\bar{X})=0$ by the isomorphisms (3). Now, by the short exact sequence

$$
0 \longrightarrow \Gamma_{\mathfrak{b}}(X) \longrightarrow X \longrightarrow \bar{X} \longrightarrow 0
$$

we get the long exact sequence

$$
\cdots \longrightarrow \mathrm{H}_{\mathfrak{a}+\mathfrak{b}}^{t}\left(\Gamma_{\mathfrak{b}}(X)\right) \longrightarrow \mathrm{H}_{\mathfrak{a}+\mathfrak{b}}^{t}(X) \longrightarrow \mathrm{H}_{\mathfrak{a}+\mathfrak{b}}^{t}(\bar{X}) \longrightarrow \cdots
$$

Since $\mathrm{H}_{\mathfrak{a}+\mathfrak{b}}^{t}\left(\Gamma_{\mathfrak{b}}(X)\right)=\mathrm{H}_{\mathfrak{a}}^{t}\left(\Gamma_{\mathfrak{b}}(X)\right)=0$, the above long exact sequence shows that $\mathrm{H}_{\mathfrak{a}+\mathfrak{b}}^{t}(X)=0$.

Lemma 2.2. Let $s$ and $t$ be non-negative integers such that

(i) $\mathrm{H}_{\mathfrak{a}}^{s+t-i}\left(\mathrm{H}_{\mathfrak{b}}^{i}(X)\right)=0$ for all $i \neq t$,

(ii) $\mathrm{H}_{\mathfrak{a}}^{s+t-i+1}\left(\mathrm{H}_{\mathfrak{b}}^{i}(X)\right)=0$ for all $i<t$, and

(iii) $\mathrm{H}_{\mathfrak{a}}^{s+t-i-1}\left(\mathrm{H}_{\mathfrak{b}}^{i}(X)\right)=0$ for all $i>t$.

Then we have the isomorphism $\mathrm{H}_{\mathfrak{a}}^{s}\left(\mathrm{H}_{\mathfrak{b}}^{t}(X)\right) \cong \mathrm{H}_{\mathfrak{a}+\mathfrak{b}}^{s+t}(X)$.

Proof. Let $\bar{X}=X / \Gamma_{\mathfrak{b}}(X)$ and $Q=\mathrm{E}_{R}(\bar{X}) / \bar{X}$ where $\mathrm{E}_{R}(\bar{X})$ is an injective hull of $\bar{X}$. We prove by using induction on $t$. In the case that $t=0$, we have $\mathrm{H}_{\mathfrak{a}+\mathfrak{b}}^{s-1}(\bar{X})=0=\mathrm{H}_{\mathfrak{a}+\mathfrak{b}}^{s}(\bar{X})$ from hypothesis (iii) and (i), and Lemma [2]. Since $\mathrm{H}_{\mathfrak{a}+\mathfrak{b}}^{s}\left(\Gamma_{\mathfrak{b}}(X)\right) \cong \mathrm{H}_{\mathfrak{a}}^{s}\left(\Gamma_{\mathfrak{b}}(X)\right)$, the assertion follows by the exact sequence

$$
\mathrm{H}_{\mathfrak{a}+\mathfrak{b}}^{s-1}(\bar{X}) \longrightarrow \mathrm{H}_{\mathfrak{a}+\mathfrak{b}}^{s}\left(\Gamma_{\mathfrak{b}}(X)\right) \longrightarrow \mathrm{H}_{\mathfrak{a}+\mathfrak{b}}^{s}(X) \longrightarrow \mathrm{H}_{\mathfrak{a}+\mathfrak{b}}^{s}(\bar{X}),
$$

obtained from the short exact sequence

$$
0 \longrightarrow \Gamma_{\mathfrak{b}}(X) \longrightarrow X \longrightarrow \bar{X} \longrightarrow 0 \text {. }
$$

Suppose that $t>0$ and that $t-1$ is settled. From the isomorphisms (四) and the assumptions, we have

- $\mathrm{H}_{\mathfrak{a}}^{s+(t-1)-i}\left(\mathrm{H}_{\mathfrak{b}}^{i}(Q)\right)=\mathrm{H}_{\mathfrak{a}}^{s+t-(i+1)}\left(\mathrm{H}_{\mathfrak{b}}^{i+1}(X)\right)=0$ for all $i \neq t-1$,

- $\mathrm{H}_{\mathfrak{a}}^{s+(t-1)+1-i}\left(\mathrm{H}_{\mathfrak{b}}^{i}(Q)\right)=\mathrm{H}_{\mathfrak{a}}^{s+t+1-(i+1)}\left(\mathrm{H}_{\mathfrak{b}}^{i+1}(X)\right)=0$ for all $i<t-1$, and

- $\mathrm{H}_{\mathfrak{a}}^{s+(t-1)-1-i}\left(\mathrm{H}_{\mathfrak{b}}^{i}(Q)\right)=\mathrm{H}_{\mathfrak{a}}^{s+t-1-(i+1)}\left(\mathrm{H}_{\mathfrak{b}}^{i+1}(X)\right)=0$ for all $i>t-1$.

Thus we get $\mathrm{H}_{\mathfrak{a}+\mathfrak{b}}^{s+(t-1)}(Q) \cong \mathrm{H}_{\mathfrak{a}}^{s}\left(\mathrm{H}_{\mathfrak{b}}^{t-1}(Q)\right)$ by the induction hypothesis on $Q$. Therefore $\mathrm{H}_{\mathfrak{a}+\mathfrak{b}}^{s+t}(\bar{X}) \cong \mathrm{H}_{\mathfrak{a}}^{s}\left(\mathrm{H}_{\mathfrak{b}}^{t}(X)\right)$ from the isomorphisms (四) and (ㅈ). On the other hand, by assumptions (i) and (ii), and the exact sequence

$$
\mathrm{H}_{\mathfrak{a}+\mathfrak{b}}^{s+t}\left(\Gamma_{\mathfrak{b}}(X)\right) \longrightarrow \mathrm{H}_{\mathfrak{a}+\mathfrak{b}}^{s+t}(X) \longrightarrow \mathrm{H}_{\mathfrak{a}+\mathfrak{b}}^{s+t}(\bar{X}) \longrightarrow \mathrm{H}_{\mathfrak{a}+\mathfrak{b}}^{s+t+1}\left(\Gamma_{\mathfrak{b}}(X)\right)
$$

obtained from the short exact sequence

$$
0 \longrightarrow \Gamma_{\mathfrak{b}}(X) \longrightarrow X \longrightarrow \bar{X} \longrightarrow 0
$$


we get $\mathrm{H}_{\mathfrak{a}+\mathfrak{b}}^{s+t}(\bar{X}) \cong \mathrm{H}_{\mathfrak{a}+\mathfrak{b}}^{s+t}(X)$. Hence $\mathrm{H}_{\mathfrak{a}}^{s}\left(\mathrm{H}_{\mathfrak{b}}^{t}(X)\right) \cong \mathrm{H}_{\mathfrak{a}+\mathfrak{b}}^{s+t}(X)$ which completes the proof.

Lemma 2.3. Let $a_{1}, \ldots, a_{n}$ be an $\mathfrak{a}$-filter regular $M$-sequence. Then, for all $0 \leq i \leq n-1$, $\operatorname{Supp}_{R}\left(\mathrm{H}_{\left(a_{1}, \ldots, a_{n}\right)}^{i}(M)\right) \subseteq \operatorname{Var}(\mathfrak{a})$. In particular,

$$
\mathrm{H}_{\mathfrak{a}}^{j}\left(\mathrm{H}_{\left(a_{1}, \ldots, a_{n}\right)}^{i}(M)\right) \cong \begin{cases}\mathrm{H}_{\left(a_{1}, \ldots, a_{n}\right)}^{i}(M) & \text { if } j=0, \\ 0 & \text { if } j>0,\end{cases}
$$

for all $0 \leq i \leq n-1$.

Proof. Let $0 \leq i \leq n-1$ and $\mathfrak{p} \in \operatorname{Supp}_{R}\left(\mathrm{H}_{\left(a_{1}, \ldots, a_{n}\right)}^{i}(M)\right)$. Assume contrarily that $\mathfrak{p} \notin \operatorname{Var}(\mathfrak{a})$. Thus $\mathfrak{p} \in \operatorname{Spec}(R) \backslash \operatorname{Var}(\mathfrak{a})$ and so $\frac{a_{1}}{1}, \ldots, \frac{a_{n}}{1}$ is a weak $M_{\mathfrak{p}}$-sequence. Hence $\mathrm{H}_{\left(\frac{a_{1}}{1}, \ldots, \frac{a_{n}}{1}\right)}^{i}\left(M_{\mathfrak{p}}\right)=$ 0 . Therefore we get $\left(\mathrm{H}_{\left(a_{1}, \ldots, a_{n}\right)}^{i}(M)\right)_{\mathfrak{p}}=0$. This contradiction shows that $\mathfrak{p} \in \operatorname{Var}(\mathfrak{a})$.

Now we are ready to give an elementary and simple proof for (प).

Proof of Nagel-Schenzel formula. Let $i<n$ (resp. $i \geq n$ ). Consider Lemma [2.3 and apply Lemma [2.2 with $s=0, t=i$, and $\mathfrak{b}=\left(a_{1}, \ldots, a_{n}\right)$ (resp. $s=i-n, t=n$, and $\left.\mathfrak{b}=\left(a_{1}, \ldots, a_{n}\right)\right)$.

\section{REFERENCES}

[1] M. P. Brodmann and R. Y. Sharp, Local Cohomology: An Algebraic Introduction with Geometric Applications, Cambridge University Press, Cambridge, 1998.

[2] W. Bruns and J. Herzog, Cohen-Macaulay Rings, Cambridge University Press, Cambridge, 1998.

[3] K. Khashyarmanesh and Sh. Salarian, Filter regular sequences and the finiteness of local cohomology modules, Comm. Algebra 26 (1998) 2483-2490.

[4] U. Nagel and P. Schenzel, Cohomological annihilators and Castelnuovo-Mumford regularity, Commutative algebra: Syzygies, multiplicities, and birational algebra, Contemp. Math. 159 (1994) 307-328.

[5] J. J. Rotman, An Introduction to Homological Algebra, Academic Press, San Diego, 1979.

[6] P. Schenzel, N. V. Trung, and N. T. Cuong, Verallgemeinerte Cohen-Macaulay-Moduln, Math. Nachr. 85 (1978) 57-73.

[7] J. Stuckrad and W. Vogel, Buchsbaum Rings and Applications, VEB Deutscher Verlag der Wissenschaften, Berlin, 1986.

\section{Alireza Vahidi}

Department of Mathematics, Payame Noor University (PNU),

P.O.BOX, 19395-4697,

Tehran, Iran.

vahidi.ar@pnu.ac.ir 